\title{
PHOTODISSOCIATION PROCESSES OF ASTROPHYSICAL MOLECULES
}

\author{
Ewine F. van Dishoeck \\ Harvard-Smithsonian Center for Astrophysics \\ 60 Garden Street \\ Cambridge, MA 02138 \\ U.S.A.
}

The photodissociation processes of molecules in various astrophysical regions are discussed, and the rates for photodissociation by the unattenuated interstellar radiation field are reviewed.

\section{INTRODUCTION}

Photodissociation has long been recognized to play an important role in various astrophysical regions. Eddington (1926) had already considered the possibility of photodissociation of a molecule in the interstellar medium, and estimated a lifetime against photodissociation of about 500 years. By accident, this estimate turns out to be of the correct order of magnitude. In cometary atmospheres, photodissociation was suggested by Wurm $(1934,1935)$ to be responsible for the formation of many of the observed radicals. More recently, photodissociation has also been shown to be important in the study of circumstellar molecules (Goldreich and Scoville 1976). In most astrophysical regions, photodissociation is the dominant destruction mechanism of the molecules. Accurate rates are therefore crucial in any model which attempts to reproduce the observed abundances of the species in these regions.

\section{PHOTODISSOCIATION CROSS SECTIONS}

Information about the basic molecular cross sections for photodissociation as functions of wavelength may be obtained both from laboratory experiments and from theory. For chemically stable non-reactive molecules such as $\mathrm{H}_{2} \mathrm{O}, \mathrm{N}_{2}, \mathrm{O}_{2}, \mathrm{CH}_{4}$, ..., measurements of low-resolution photoabsorption cross sections are available over a wide range of wavelengths. Reviews have been given by Hudson (1971), Okabe (1978), Huebner and Carpenter (1979) and Lee (1984). The absorption of the photons may result in re-emission, dissociation or ionization of the molecule, and most absorption measurements do not distinguish between these various processes. Additional information on the emission and ionization cross sections of the molecules is therefore needed to infer the photodissociation cross sections. For absorption which is continuous as a function of wavelength, the cross sections 
obtained at low resolution generally agree well with more recent high-resolution measurements. For discrete absorptions, however, measurements which are performed at relatively high pressures and which do not resolve individual lines, may underestimate the absorption cross sections by orders of magnitude. A related experimental problem is the fact that most molecules of astrophysical interest have large cross sections for photodissociation at vacuum ultraviolet (VUV) wavelengths, where there are still no intense light sources commonly available for highresolution spectroscopy. Measurements using VUV lasers are generally limited to only a few specific wavelengths. Synchrotron radiation sources cover a wider wavelength range and provide an intense almost featureless background continuum for the absorption measurements, but until recently no photoelectric VUV spectrographs with sufficiently high resolution were available at these facilities. Therefore, even for many stable molecules no high-resolution measurements exist at short wavelengths $\lambda<1100 \AA$. Experimental data on unstable reactive species such as the radicals $\mathrm{CH}, \mathrm{OH}, \mathrm{C}_{2} \mathrm{H}$, which are widely observed in various astrophysical regions, are very limited, and few reliable quantitative experiments exist.

Theory can also provide important information on photodissociation processes, especially for molecules which are difficult to prepare in the laboratory. $\mathrm{Ab}$ initio quantum chemical calculations of potential energy curves and/or surfaces of excited electronic states, together with correlation diagrams, give insight into the possible photodissociation pathways of the molecule. Computations of the transition dipole moments connecting the excited electronic states with the ground state yield absorption and photodissociation cross sections. Reliable quantitative theoretical results -i.e., transition energies accurate to better than $0.2 \mathrm{eV}$ and oscillator strengths to better than $30 \%$ - may be obtained for small molecules with the number of (active) electrons limited to about 20. In practice, accurate calculations are restricted to the lowest four to five excited electronic states of a given molecular symmetry. For many molecules of astrophysical interest, this range includes the most important photodissociation paths.

\section{PHOTODISSOCIATION PROCESSES}

Photodissociation of a molecule may take place in several ways. Figs. 1a-d illustrate the various processes for a diatomic molecule, where the only vibrational degree of freedom is the internuclear distance. For polyatomic molecules, the mechanisms are similar but more complicated, because the potential energy surfaces are multi-dimensional. The simplest dissociation process is by direct absorption from the ground electronic state into the repulsive part of an excited electronic state, as shown in Fig. 1a. Since spontaneous emission back into the lower state is a comparatively slow process, all absorptions will lead to dissociation of the molecule. The photodissociation of the $\mathrm{CH}^{+}$and $\mathrm{NH}$ molecules, for example, proceeds mainly in this way. The photodissociation cross section is continuous as a function of wavelength and has its maximum value at the vertical excitation energy (indicated by the arrow in Fig. 1a). Dissociation may also occur in indirect ways following discrete absorptions into bound electronic states. The bound levels of the excited state may couple through a radiationless process with the continuum of a final dissociating state of a different symmetry. This process of predissociation dominates the photodissociation of the $\mathrm{CO}$ and $\mathrm{HCl}$ molecules, for example, and is illustrated in Fig. 1b. The spectrum consists in this case of a series of discrete peaks which 


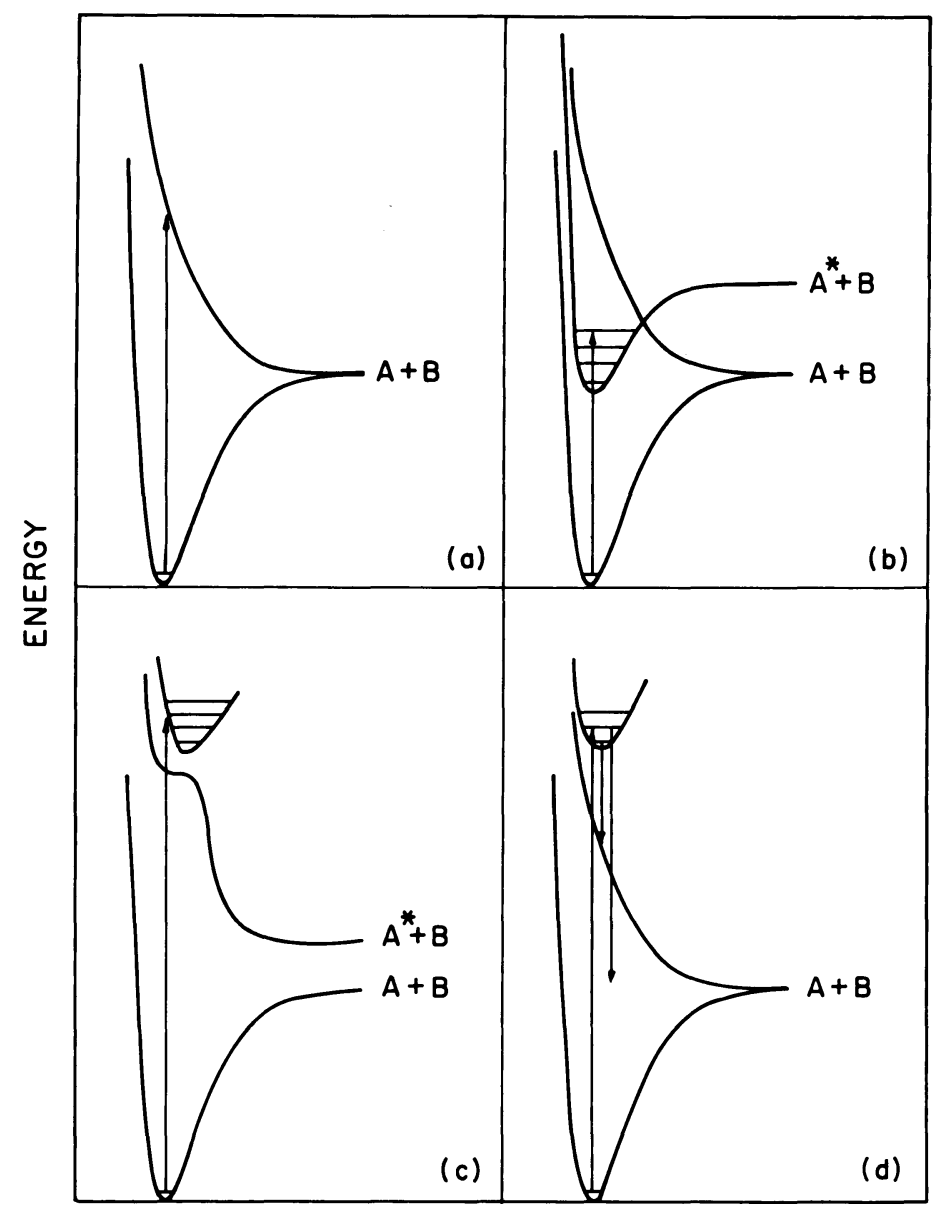

R

Figure 1. Potential energy curves as functions of internuclear distance $R$ illustrating the processes of direct photodissociation (a), predissociation (b), photodissociation through coupled states (c) and spontaneous radiative dissociation (d) for a diatomic molecule $A B$.

are broadened by predissociation. The bound excited levels can also interact with the continuum of a repulsive state of the same symmetry, as indicated in Fig. 1c. This coupled states process plays an important role in the photodissociation of the $\mathrm{CH}$ and $\mathrm{OH}$ molecules, for example. The details of the spectrum depend on the properties of the molecule considered. It may consist of a broad continuous background with a superposed series of resonances having asymmetrical line shapes. Finally, absorptions into bound electronic states may be followed by spontaneous emission into the continuum of a lower-lying state, as illustrated in Fig. 1d. The photodissociation of the important $\mathrm{H}_{2}$ molecule proceeds by this mechanism of spontaneous radiative dissociation. In this case, the spectrum contains a series of sharp discrete peaks. 
Note that although the lowest threshold for photodissociation lies at the energy for dissociation of the molecule into ground-state products, the maximum of the photodissociation cross section generally occurs at higher energies.

The cross sections for isotopic species will be very similar to those for the main species if the photodissociation of the molecule is dominated by the direct mechanism. For processes proceeding through line absorption, however, substantial differences may occur if the onset of predissociation of the isotopic species starts at a different energy level, with e.g. a lower oscillator strength, than that of the principal species.

The models of the various astrophysical regions also require information about the products of the photodissociation processes. Photodissociation of a diatomic molecule may result not only in ground state atoms, but also in excited atoms from which the emission may be observed. A polyatomic molecule may photodissociate into different fragments at different wavelengths, and each of the molecular fragments may be electronically, vibrationally and rotationally excited. Little reliable experimental and theoretical information is available on these problems.

\section{PHOTODISSOCIATION RATES}

The rate of photodissociation $k_{p d}$ of a molecule by absorption from a lower level 1 into a continuous upper channel $u$ is given by

$$
k_{p d}^{c o n t}=\int \sigma_{u l}(\lambda) x_{l} I(\lambda) d \lambda \mathrm{s}^{-1},
$$

where $\sigma$ is the cross section for photodissociation in $\mathrm{cm}^{2}, x_{l}$ is the fractional population in level 1 , and $I$ is the mean intensity of the radiation measured in photons $\mathrm{cm}^{-2} \mathrm{~s}^{-1} \AA^{-1}$ as function of wavelength $\lambda$ in $\AA$. For indirect processes, the rate of dissociation by absorption into an individual level of a bound upper state $u$ from the lower level $l$ is

$$
k_{p d}^{l i n e}=\frac{\pi e^{2}}{m c^{2}} \lambda_{u l}^{2} f_{u l} \eta_{u} x_{l} I\left(\lambda_{u l}\right) \mathrm{s}^{-1},
$$

where $f_{u l}$ is the line absorption oscillator strength and $\eta_{u}$ is the dissociation efficiency of the upper state level, which lies between zero and unity. The value of $\eta_{u}$ is related to molecular quantities in a complicated way, and is often not well known. The numerical value of the factor $\pi e^{2} / m c^{2}$ in Eq.(2) is $8.85 \times 10^{-21}$ in the employed units. The total photodissociation rate of a molecule is obtained by summation over all possible channels. Since the variation of the intensity of the radiation with wavelength is substantially different in, for example, interstellar clouds and cometary atmospheres, different photodissociation channels may be important in different regions. In the following, the various astrophysical regions will be discussed separately.

\subsection{Interstellar photodissociation}

The intensity of the mean unattenuated interstellar radiation field $I^{\circ}(\lambda)$ at ultraviolet wavelengths has been discussed e.g. by Habing (1968) and Draine (1978). At 
longer wavelengths, it has been estimated by Witt and Johnson (1973), Jura (1979) and Mathis et al. (1983). The intensity decreases with shorter wavelengths and vanishes below $912 \AA(13.6 \mathrm{eV})$ due to absorptions by atomic hydrogen. Lowerlying channels are therefore relatively more effective than higher-excited states in the destruction of the interstellar molecules. The intensity can be represented by

$$
\begin{aligned}
I^{o}(\lambda) & =3.24 \times 10^{15} \lambda^{-3}-5.13 \times 10^{18} \lambda^{-4}+2.03 \times 10^{21} \lambda^{-5} \text { for } \lambda<2000 \AA \\
& =7.32 \times 10^{2} \lambda^{0.7} \text { for } \lambda>2000 \AA .
\end{aligned}
$$

Inside interstellar clouds, the intensity of the radiation field is reduced by several factors. The grains present in the cloud will scatter and absorb the photons, particularly those with shorter wavelengths. The amount of continuum attenuation at each depth into the cloud depends upon the extinction, the albedo and the scattering phase function of the grains (see e.g. Sandell and Mattila 1975, Roberge et al. 1981), none of which is known accurately. Current models of interstellar grains (Draine and Lee 1984, Chlewicki and Greenberg 1984) favor an albedo $\omega_{\lambda} \simeq 0.4$ and a scattering asymmetry parameter $\mathrm{g}_{\lambda} \simeq 0.6-0.9$ for $\lambda<1500 \AA$. Furthermore, if the photodissociation of a molecule is dominated by indirect processes through line absorption, and if its abundance in the cloud is sufficiently high, the rate is affected by self-shielding. Finally, the rates of molecules for which the photodissociation occurs mainly at $\lambda<1100 \AA$ may be further reduced by the opacity due to molecular hydrogen and atomic carbon.

In diffuse clouds and the outer parts of thicker clouds, photodissociation is the dominant destruction mechanism of the neutral molecules. Molecular ions are generally more rapidly destroyed by dissociative recombination with electrons and/or reactions with the most abundant neutral species. However, photodissociation may be important for those ions for which the above reactions are slow, or in cases where the degree of ionization is low, such as certain magnetohydrodynamical shocks (Pineau des Forêts et al. 1986).

Most models of dense molecular clouds have assumed that photodissociation is negligible deep inside the clouds. Prasad and Tarafdar (1983), however, have suggested that UV photons may be generated inside clouds by the interaction of cosmic rays with molecular hydrogen. The number of photons formed by this mechanism may be large enough for the photodissociation rates to be comparable to other destruction rates.

In interstellar regions subjected to fast shocks, the radiation field is expected to have a high intensity at $1215.16 \AA$ because of recombination and collisional excitation of atomic hydrogen leading to Lyman alpha radiation (Hollenbach and McKee 1979). It is thus important to know whether a molecule has a photodissociation channel at this wavelength.

With the assumption that the attenuation in the lines and continuum are separable, the photodissociation rate of molecule $\mathbf{X}$ through line absorption inside an interstellar cloud may be written

$$
k_{p d}=\sum_{u, l} \frac{\pi e^{2}}{m c^{2}} \lambda_{u l}^{2} f_{u l} \eta_{u} x_{l} I^{o}\left(\lambda_{u l}\right) \theta_{u l}\left(N_{l}(X)\right) \theta_{c}\left(A_{V}\right)
$$

where $\theta_{c}\left(A_{V}\right)$ is the continuum attenuation at a depth characterized by a visual extinction $A_{V}$. The line shielding function $\theta_{u l}$ depends on the lower state column 
density $N_{l}$ of species $\mathrm{X}$ at that depth inside the cloud, and has been discussed by Hollenbach et al. (1971) and Federman et al. (1979). It may be expressed by

$$
\theta_{u l}\left(N_{l}\right)=\frac{d W_{u l}}{d N_{l}} / \frac{\pi e^{2}}{m c^{2}} f_{u l} \lambda_{u l}^{2},
$$

where $W_{u l}$ is the equivalent width of the line in wavelength units. Standard subroutines exist for computing a curve-of-growth (see e.g. Rodgers and Williams 1974) and the differentiation in (5) can be performed numerically.

The photodissociation rates of various molecules by the unattenuated interstellar radiation field (Eq.(3)) are summarized in Table 1. It is supposed that the molecules are initially in the lowest vibrational and rotational level of the ground electronic state, unless otherwise indicated. The upper part of the table contains those molecules for which the photodissociation processes are either accurately measured or well understood from theory, so that the resulting photodissociation rate is known to better than 50\%. The second part includes those species for which some photodissociation channels are known, leading to a lower limit to the rate, but for which information on additional photodissociation processes is lacking. It also includes those molecules for which photodissociation occurs largely through line absorption and for which the experimental values are uncertain. The rates in this category are known to within a factor of about two. The lowest part of the table contains a few examples of important molecules for which still very little is known about the photodissociation. The table also includes - where possiblefor comparison the rates used by Black and Dalgarno (1977), since this is still the most widely referenced source for photodissociation rates. In general, the new rates are significantly larger. Part of the increases stem from the fact that the employed radiation field (Eq.(3)) is about a factor two stronger in the UV than that used by Black and Dalgarno (1977). Because of space limitations, only a few comments on the basic photodissociation processes and cross sections that were used in the calculations can be made in the following. More details will be given in a future paper (van Dishoeck, in preparation).

Photodissociation of the $\mathrm{H}_{2}$ molecule proceeds by absorptions of UV photons in the Lyman, $\mathrm{B}^{1} \Sigma_{\mathbf{u}}^{+}-\mathrm{X}^{1} \Sigma_{\mathrm{g}}^{+}$, and Werner, $\mathrm{C}^{1} \Pi_{\mathbf{u}}-\mathrm{X}^{1} \Sigma_{\mathbf{g}}^{+}$, systems in the 912$1100 \AA$ range. The absorptions are followed by fluorescence to the vibrational continuum of the ground state (Solomon 1965, Stecher and Williams 1967) with probabilities $\eta_{u}$ calculated by Stephens and Dalgarno (1972). Oscillator strengths for the transitions have been presented by Allison and Dalgarno (1970). The photodissociation rate in the unattenuated radiation field can be calculated by summing over all lines according to Eq.(2), and is about $7.2 \times 10^{-11} \mathrm{~s}^{-1}$ under typical interstellar conditions.

In clouds which have a total $\mathrm{H}_{2}$ column density larger than $10^{14} \mathrm{~cm}^{-2}$, selfshielding becomes important. A proper calculation of the line-shielding function $\theta_{u l}$ requires a simultaneous treatment of the abundance and excitation of $\mathrm{H}_{2}$ as functions of depth. For applications in which the $\mathrm{H}_{2}$ population distribution is not required and in which the fine details in the depth dependence of the absorption rate need not be described rigorously, a simpler method may be used. In this approximation, the summation in Eq.(4) is replaced by a shielding function for a single line and an appropriate scaling factor $\mathcal{N}$ required to give the correct 
TABLE 1

Photodissociation rates $k_{p d}^{o}\left(\right.$ in $\mathrm{s}^{-1}$ ) of various molecules by the unattenuated interstellar radiation field (cf. Eq.(3)) ${ }^{a}$

\begin{tabular}{|c|c|c|c|}
\hline Species & $\begin{array}{l}\text { Lower } \\
\text { Limit }\end{array}$ & $\begin{array}{c}\text { Best } \\
\text { Estimate }\end{array}$ & $\begin{array}{c}\mathrm{BD}^{b} \\
(1977)\end{array}$ \\
\hline $\mathrm{H}_{2}$ & $\ldots$ & $7.2(-11)^{c}$ & $\ldots$ \\
\hline $\mathrm{O}_{2}$ & $\ldots$ & $7.9(-10)^{d}$ & $3.3(-10)$ \\
\hline $\mathrm{H}_{2} \mathrm{O}$ & $\ldots$ & $8.0(-10)$ & $3.2(-10)$ \\
\hline $\mathrm{CH}_{4}$ & $\ldots$ & $1.1(-9)$ & $8.0(-10)$ \\
\hline $\mathrm{NH}_{3}$ & $\ldots$ & $1.1(-9)$ & $5.5(-10)$ \\
\hline $\mathrm{HCN}$ & $\ldots$ & $1.5(-9)$ & $1.0(-10)$ \\
\hline $\mathrm{HCl}$ & $\ldots$ & $9.0(-10)$ & $1.3(-10)$ \\
\hline $\mathrm{OH}$ & $\ldots$ & $4.2(-10)$ & $1.5(-10)$ \\
\hline $\mathrm{NaH}$ & $\ldots$ & $7.0(-9)$ & ... \\
\hline $\mathrm{CH}^{+}$ & $\ldots$ & $3.2(-10)$ & $4.6(-12)$ \\
\hline $\mathrm{SiH}^{+}$ & $\ldots$ & $2.2(-9)$ & ... \\
\hline $\mathrm{OH}^{+}$ & $\ldots$ & $<1.0(-12)$ & $1.2(-12)$ \\
\hline $\mathrm{H}_{3}^{+}$ & $\ldots$ & $<1.0(-12)$ & $2.8(-8)$ \\
\hline$\overline{\mathrm{CH}}$ & $8.0(-10)$ & $1.2(-9)$ & $1.4(-10)$ \\
\hline $\mathrm{NH}$ & $3.7(-10)$ & $5.0(-10)$ & $8.0(-10)$ \\
\hline $\mathrm{SiH}$ & $1.5(-9)$ & $3.0(-9)$ & ... \\
\hline $\mathrm{C}_{2}$ & $1.8(-10)$ & $2.3(-10)$ & $5.0(-11)$ \\
\hline CN & $1.5(-10)$ & $3.0(-10)$ & $5.0(-11)$ \\
\hline $\mathrm{CH}_{2}$ & $4.9(-10)$ & $7.0(-10)$ & $5.0(-11)$ \\
\hline $\mathrm{NH}_{2}$ & $5.8(-10)$ & $8.0(-10)$ & $\ldots$ \\
\hline No & $\ldots$ & $4.0(-10)$ & $\ldots$ \\
\hline $\mathrm{C}_{2} \mathrm{H}_{2}$ & $\ldots$ & $3.2(-9)$ & $\ldots$ \\
\hline $\mathrm{HC}_{3} \mathrm{~N}$ & $\ldots$ & $5.6(-9)$ & $\ldots$ \\
\hline $\mathrm{CH}_{3} \mathrm{CN}$ & $\ldots$ & $2.4(-9)$ & $\ldots$ \\
\hline$\overline{\mathrm{CO}}$ & & $7.0(-11)$ & $5.0(-12)$ \\
\hline $\mathrm{N}_{2}$ & $5.0(-11)$ & $2.3(-10)$ & ... \\
\hline $\mathrm{C}_{2} \mathrm{H}$ & $2.0(-10)$ & $5.0(-10)$ & $1.4(-10)$ \\
\hline $\mathrm{C}_{3}$ & $\ldots$ & $2.0(-9)$ & $\ldots$ \\
\hline $\mathrm{MgH}$ & $3.3(-11)$ & $5.0(-10)$ & $\ldots$ \\
\hline
\end{tabular}

a The first part of the table contains those molecules for which the uncertainty in the rate is less than $50 \%$; the second part those molecules for which the uncertainty is between $50 \%$ and a factor of about two; the last part some species for which the uncertainty is at least a factor two and probably an order of magnitude. ${ }^{b}$ Black and Dalgarno (1977). ${ }^{c}$ see text. ${ }^{d}$ The notation $1.0(-9)$ indicates $1.0 \times 10^{-9}$. 
absolute value of the rate. Comparison with extensive calculations of the $\mathrm{H}_{2}$ photodissociation rate as described by van Dishoeck and Black (1986) suggests that the rate can be well approximated using $\mathcal{N}=135$ and an effective line at $\lambda=1000 \AA$ with an oscillator strength $f_{\text {eff }}=3.5 \times 10^{-3}$, an inverse lifetime of $5.7 \times 10^{8} \mathrm{~s}^{-1}$ and a dissociation efficiency $\eta_{\text {eff }}=0.127$.

The photodissociation of $\mathrm{O}_{2}$ proceeds mainly by absorption into the SchumannRunge continuum and the predissociated Schumann-Runge bands. The rate in interstellar clouds has been discussed by Black and Smith (1984).

The photodissociation rates of the $\mathrm{H}_{2} \mathrm{O}, \mathrm{CH}_{4}, \mathrm{NH}_{3}$, and $\mathrm{HCN}$ molecules are based on experimental cross sections which have been reviewed recently by Lee (1984). Information on the products of the photodissociation processes can be found in the same paper. Photodissociation of the $\mathrm{C}_{2} \mathrm{H}_{2}$ and NO molecules apparently proceeds mainly through discrete absorptions for which the experimental cross sections (Lee 1984) may be more uncertain.

$\mathrm{HCl}$ is one of the few molecules for which both reliable experimental (Inn 1975, Smith et al. 1980) and theoretical (van Dishoeck et al. 1982) cross sections are available. The agreement is satisfactory. The larger photodissociation rate helps explain why $\mathrm{HCl}$ has not yet been observed in diffuse interstellar clouds.

The photodissociation processes in the $\mathrm{OH}$ molecule have been discussed extensively by van Dishoeck and Dalgarno $(1983,1984 \mathrm{a})$. Recent experiments (Nee and Lee 1985) have suggested a somewhat larger rate than listed in the table. However, the uncertainty in the measured values is large, so that the theoretical values are preferable.

Photodissociation of the metal hydride $\mathrm{NaH}$ is very rapid, as the calculations by Kirby and Dalgarno (1978) indicate. On the other hand, the calculated cross sections for the photodissociation of $\mathrm{MgH}$ through the lowest two excited states are very small (Kirby et al. 1979). The $\mathrm{MgH}$ photodissociation rate may be increased significantly, however, by possible contributions from higher excited states.

The photodissociation of the interstellar $\mathrm{CH}^{+}$ion has been discussed thoroughly by Kirby et al. (1980). Photodissociation occurs mainly by absorptions from the ground state into high-lying excited singlet states. Photodissociation by absorption into the first excited $\mathrm{A}^{1} \Pi$ state has been calculated most recently by Graff and Moseley (1984). This cross section is small in interstellar clouds compared with other channels, and may be neglected. In contrast, the cross section for photodissociation through the $\mathrm{A}^{1} \Pi$ state of the isovalent $\mathrm{SiH}^{+}$ion is substantial (K. Kirby and P.D. Singh, priv. comm.), resulting in an order of magnitude larger photodissociation rate than for $\mathrm{CH}^{+}$.

The photodissociation processes in the $\mathrm{OH}^{+}$ion have been investigated recently through quantum chemical calculations by Saxon and Liu (1985). The interstellar rate is very low because all important photodissociation channels have excitation energies in excess of $13.6 \mathrm{eV}$. The photodissociation rate of the interstellar $\mathrm{H}_{3}^{+}$ion is very small for similar reasons (Kulander and Bottcher 1978). The previously suggested large $\mathrm{H}_{3}^{+}$photodissociation rate was based on incorrect arguments.

The photodissociation of $\mathrm{CH}$ in interstellar clouds has been discussed by Solomon and Klemperer (1972), and Barsuhn and Nesbet (1978). The processes have been reexamined through quantum chemical calculations by van Dishoeck (1986). The summed photodissociation rate for the states considered in these calculations is about $8 \times 10^{-10} \mathrm{~s}^{-1}$. The uncertainty in the rate stems from the fact 
that $\mathrm{CH}$ has a comparatively low-lying first ionization potential at $10.64 \mathrm{eV}$ (1165 $\AA)$. Barsuhn and Nesbet suggested that many of the Rydberg states converging to this and higher ionization limits contribute significantly to the $\mathrm{CH}$ photodissociation, and possibly increase the rate to $1.6 \times 10^{-9} \mathrm{~s}^{-1}$. It is not clear, however, whether the absorptions into states above the ionization threshold will lead to dissociation or ionization of the molecule. The calculations by van Dishoeck (1986) confirm the conclusion of Giusti-Suzor and Lefebvre-Brion (1977) that the CH repulsive potentials do not cross the Franck-Condon region of the $\mathrm{CH}^{+}$ground state, suggesting that the absorptions lead to ionization. It also indicates that the $\mathrm{CH}^{+}$dissociative recombination rate may be slow at interstellar temperatures. The photodissociation rate of the isovalent $\mathrm{SiH}$ molecule listed in the table is estimated from excitation energies and oscillator strengths calculated by Lewerenz et al. (1983). This rate is affected by similar uncertainties.

The lower limit to the photodissociation rate of interstellar $\mathrm{NH}$ is based on potential curves calculated by Goldfield and Kirby (1986) and S.R. Langhoff (priv. comm.), together with transition dipole moments reported by Marian and Klotz (1985). The rate may be increased by contributions from higher-lying states, but it is unlikely to be as large as the value guessed by Black and Dalgarno (1977).

Cross sections for the photodissociation of $\mathrm{C}_{2}$ have been computed by Pouilly et al. (1983). Because of the limited size of the calculations and possible contributions from higher-lying states, the rate is accurate to about $50 \%$.

Calculations on the photodissociation of the $\mathrm{CN}$ molecule have been reported by Lavendy et al. (1984). A large rate of $10^{-9} \mathrm{~s}^{-1}$ has been suggested. However, the calculations may be of limited accuracy, particularly for the higher excited states. More extensive calculations by van Dishoeck (1986) indicate the lower rate listed in the table. All photodissociations occur at wavelengths shorter than $1050 \AA$, in agreement with the fact that no $\mathrm{CN}$ photodissociation is observed experimentally for $\lambda>1060 \AA$ (Nee and Lee 1985).

The photodissociation processes of the $\mathrm{CH}_{2}$ molecule have not been discussed in detail before. The rate given in the table is based on excitation energies and transition dipole moments calculated by Römelt et al. (1981), together with considerations of correlation diagrams. The summed rate by absorptions into these states is quite substantial and may be increased further by higher-lying states. As for the $\mathrm{CH}$ molecule, the ionization potential of $\mathrm{CH}_{2}$ lies comparatively low in energy, around $10.3 \mathrm{eV}$, so that photoionization may be efficient.

Cross sections for photodissociation of $\mathrm{NH}_{2}$ through low-lying channels for use in cometary atmospheres have been reported by Saxon et al. (1982). In the interstellar medium, higher-lying channels may be important as well, and may increase the rate.

The photodissociation rates of the larger molecules $\mathrm{HC}_{3} \mathrm{~N}$ and $\mathrm{CH}_{3} \mathrm{CN}$ are based on measurements by Connors et al. (1974), Nuth and Glicker (1982) and Suto and Lee (1985).

Photodissociation of $\mathrm{N}_{2}$ appears to be possible only at the shortest wavelengths $\lambda<1000 \AA$, by absorptions into bound excited states which are probably predissociated. Oscillator strengths for these transitions were taken from Carter (1972) and may be quite uncertain.

There has been no discussion of the $\mathrm{C}_{2} \mathrm{H}$ photodissociation processes. The lower limit to the rate listed in the table is based on excitation energies and oscillator strengths calculated by Shih et al. $(1977,1979)$. The computed values may be uncertain by a factor two. The ionization threshold lies at about $11 \mathrm{eV}$. 
Higher excited states lying below and above this limit may contribute effectively to the photodissociation rate. The large rate for $\mathrm{C}_{3}$ given in the table is obtained by assuming that the states calculated by Römelt et al. (1978) contribute efficiently to photodissociation.

$\mathrm{CO}$ is the most important molecule in the category of species for which large uncertainties still exist in the photodissociation mechanisms. Experimental work has revealed no continuous absorption of CO longward of $1060 \AA$ (Lee and Guest 1981), and it is likely that there is no appreciable continuous dissociation channel longward of $912 \AA$ (Fock et al. 1980, Rostas 1985). Thus the interstellar photodissociation of $\mathrm{CO}$ is dominated by discrete absorptions into excited states, but neither the states that participate in the dissociation process nor the oscillator strengths for the transitions are known. Previous estimates (van Dishoeck 1984, Glassgold et al. 1985) assumed that the discrete photodissociation of CO proceeds mainly by absorptions into the $v^{\prime}=0$ level of the $\mathrm{E}^{1} \Pi$ state and the $v^{\prime}=1$ level of the $C^{1} \Sigma^{+}$state at 1076 and $1063 \AA$, respectively, for which large predissociation probabilities had been suggested by Lee and Guest (1981). Subsequent experiments at higher resolution (Rostas 1985, Klopotek and Vidal 1985), however, indicate that rapid predissociations in the $\mathrm{C}$ and $\mathrm{E}$ states occur only for the higher rotational levels which are not populated in interstellar clouds, although a small predissociation probability for the lower $J$ levels cannot be excluded. Photodissociation via the $\mathrm{C}$ and $\mathrm{E}$ states thus appears to be unimportant. The photodissociation of $\mathrm{CO}$ is then dominated by absorptions into higher-lying states, which are mainly Rydberg in character and which are strongly predissociated for all $J$ levels (Rostas 1985). The experiments by Fock et al. (1980) at low resolution indicate a total integrated cross section for these states of $2 \times 10^{-15} \mathrm{~cm}^{2} \AA$, and a similar number is obtained from recent spectra at higher resolution (Rostas 1985). An estimate of the summed oscillator strengths for the Rydberg transitions from the CO ionization cross sections at threshold gives $1.5 \times 10^{-15} \mathrm{~cm}^{2} \AA$. The corresponding unattenuated photodissociation rate is about $2 \times 10^{-10} \mathrm{~s}^{-1}$. Since the various experimental determinations of oscillator strengths even for some of the lower transitions differ by a factor five, the above rate is equally uncertain. The rate listed in the table is at the lower limit of this range. Further information on the oscillator strengths of individual transitions and on the predissociation probabilities is needed to calculate the shielding in the lines of $\mathrm{CO}$ and its isotopic species as a function of depth into the cloud.

\subsection{Circumstellar photodissociation}

In circumstellar shells, the radiation which is responsible for the destruction of the molecules may come either from the star itself or it may be the external interstellar radiation field (Glassgold and Huggins 1986). In the latter case, the radiation from the star is either weak or completelyshielded by dust, as is the case for the wellstudied envelope IRC+10216. The photodissociation rates of the circumstellar species will then not be significantly different from the corresponding interstellar rates summarized in Table 1 . The depth dependence of the rate is again affected by the continuum attenuation due to grains and by self-shielding. The UV scattering properties of circumstellar dust grains are particularly uncertain.

If the radiation from the star is not shielded by dust, it may easily dominate the destruction of the molecules close to the star. For example, the photodissociation rates by the chromospheric UV radiation field of the star $\alpha$ Ori are estimated 
to be about $10^{6}$ times larger than those by the interstellar radiation field (Clegg et al. 1983, see also Glassgold, this volume), so that molecule destruction close to the star is greatly enhanced. At larger radii, the interstellar radiation may again dominate.

An interesting feature of circumstellar shells compared with interstellar clouds is that the observations provide information about the spatial distribution of the molecules. This has led to photochemical models of the outer parts of circumstellar shells in which a parent molecule photodissociates into a radical, etc. Examples include $\mathrm{H}_{2} \mathrm{O} \rightarrow \mathrm{OH} \rightarrow \mathrm{O}$ (Goldreich and Scoville 1976), $\mathrm{HCN} \rightarrow \mathrm{CN}$ (Wootten et al. 1982), and $\mathrm{C}_{2} \mathrm{H}_{2} \rightarrow \mathrm{C}_{2} \mathrm{H} \rightarrow \mathrm{C}_{2} \rightarrow \mathrm{C} \rightarrow \mathrm{C}^{+}$(Huggins and Glassgold 1982). The fact that the $\mathrm{CO}$ molecule can be observed out to large distances has been interpreted to be the consequence of self-shielding (Morris and Jura 1983).

\subsection{Cometary photodissociation}

The radiation that destroys molecules in cometary atmospheres is provided by the Sun. The intensity is thus strongest at visible wavelengths, and drops very rapidly in the UV. For example, the average solar radiation intensity at $5000 \AA$ is a factor of about $10^{5}$ larger than that at $1500 \AA$. Therefore any channel which lies very low in energy may dominate the photodissociation of the molecule in cometary atmospheres, even if it has a comparatively small cross section. The same channel may be completely negligible in the interstellar medium. In addition, there are a few resonance lines at shorter wavelengths, such as the Lyman alpha line at 1215.16 $\AA$, which may be effective in the photodissociation of cometary molecules.

The calculation of the photodissociation rates in cometary atmospheres is complicated by several factors. If the photodissociation occurs by discrete absorptions in a wavelength region where the intensity of the solar radiation field is varying rapidly with wavelength, the rate may depend on the heliocentric radial velocity of the comet. This phenomenon has been demonstrated for the $\mathrm{OH}$ molecule (Jackson 1980, van Dishoeck and Dalgarno 1984b) and variations up to $50 \%$ occur in the rate. A similar process may occur for $\mathrm{CH}, \mathrm{NH}, \ldots$ Furthermore, the solar flux in the ultraviolet may vary by a factor of two or more between solar minimum and maximum (Oppenheimer and Downey 1980). Any comparison between calculations and observations should take the above factors into account.

Also for cometary atmospheres, information about the spatial distribution of the molecules may be obtained. Through detailed models of the cometary coma (Haser 1957, Festou 1981), lifetimes of the observed species may be inferred, which may be compared with theory. The photodissociation rates of molecules in cometary atmospheres have been considered by Potter and Del Ducca (1964) and Huebner and Carpenter (1979), and will be reviewed by Huebner et al. (1986).

In summary, substantial progress has been made in our understanding of the photodissociation processes in several important astrophysical molecules. Nevertheless, the cross sections at the shorter wavelengths remain uncertain for many species. Also, the information on the products of the photodissociation processes of polyatomic molecules is still very scarce. For many molecules, such as $\mathrm{HCO}, \mathrm{C}_{3}$, $\mathrm{CH}_{3}, \mathrm{CH}_{2}^{+}, \mathrm{CH}_{3}^{+}, \mathrm{H}_{2} \mathrm{O}^{+}, \mathrm{HCl}^{+}, \ldots$, almost no information is available on possible photodissociation channels. Substantial theoretical and experimental efforts are needed to remove these uncertainties in the near future. 
The author is indebted to J.H. Black for many useful discussions and to A. Dalgarno for a critical reading of the manuscript.

\section{REFERENCES}

Allison, A.C. and Dalgarno, A. 1970, Atomic Data, 1, 289.

Barsuhn, J. and Nesbet, R.K. 1978, J. Chem. Phys., 68, 2783.

Black, J.H. and Dalgarno, A. 1977, Ap. J. Suppl., 34, 405.

Black, J.H. and Smith, P.L. 1984, Ap. J., 277, 562.

Carter, V.L. 1972, J. Chem. Phys., 56, 4195.

Chlewicki, G. and Greenberg, J.M. 1984, M. N. R. A. S., 210, 791; 211, 719.

Clegg, R.E.S., van Ijzendoorn, L.J. and Allamandola, L.J. 1983, M. N. R. A. S., $203,125$.

Connors, R.E., Roebbel, J.L. and Weiss, K. 1974, J. Chem. Phys., 60, 5011.

Draine, B.T. 1978, Ap. J. Suppl., 36, 595.

Draine, B.T. and Lee, H.M. 1984, Ap. J., 285, 89.

Eddington, A.S. 1926, Proc. Roy. Soc. A, 111, 424.

Federman, S.R., Glassgold, A.E. and Kwan, J. 1979, Ap. J., 227, 466.

Festou, M.C. 1981, Astr. Ap., 95, 69.

Fock, J.-H., Gürtler, P. and Koch, E.E. 1980, Chem. Phys., 47, 87.

Giusti-Suzor, A. and Lefebvre-Brion, H. 1977, Ap. J. (Letters), 214, L101.

Glassgold, A.E. and Huggins, P.J. 1986, to appear in "The $M, S$ and $C$ stars", eds. H.R. Johnson and F. Querci (NASA, CNRS).

Glassgold, A.E., Huggins, P.J. and Langer, W.D. 1985, Ap. J., 290, 615.

Goldfield, E. and Kirby, K. 1986, in preparation.

Goldreich, P. and Scoville, N. 1976, Ap. J., 205, 144.

Graff, M.M. and Moseley, J.T. 1984, Chem. Phys. Lett., 105, 163.

Habing, H.J. 1968, Bull. Astr. Inst. Neth., 19, 421.

Haser, L. 1957, Bull. Acad. Roy. Sci. Belgique, 43, 740.

Hollenbach, D.J. and McKee, C.F. 1979, Ap. J. Suppl., 41, 555.

Hollenbach, D.J., Werner, M.W. and Salpeter, E.E. 1971, Ap. J., 163, 165.

Hudson, R.D. 1971, Rev. of Geophys. and Space Physics, 9, 305.

Huebner, W.F. and Carpenter, C.W. 1979, Los Alamos National Laboratory Informal Report LA-8085-MS.

Huebner, W.F. et al. 1986, in preparation.

Huggins, P.J. and Glassgold, A.E. 1982, Ap. J., 252, 201.

Inn, E.C.Y. 1975, J. Atmos. Sci., 32, 2375.

Jackson, W.M. 1980, Icarus, 41, 147.

Jura, M. 1979, Astrophys. Letters, 20, 89.

Kirby, K. and Dalgarno, A. 1978, Ap. J., 224, 444.

Kirby, K., Saxon, R.P. and Liu, B. 1979, Ap. J., 231, 637.

Kirby, K., Roberge, W.G., Saxon, R.P. and Liu, B. 1980, Ap. J., 239, 855.

Klopotek, P. and Vidal, C.R. 1985, J. Opt. Soc. Am. B, $2,869$.

Kulander, K. and Bottcher, C. 1978, Chem. Phys., 29, 141.

Lavendy, H., Gandara, G. and Robbe, J.M. 1984, J. Mol. Spectrosc., 106, 395.

Lee, L.C. 1984, Ap. J., $282,172$.

Lee, L.C. and Guest, J.A. 1981, J. Phys. B, 14, 3415. 
Lewerenz, M., Bruna, P.J., Peyerimhoff, S.D. and Buenker, R.J. 1983, Mol. Phys., 49, 1 .

Marian, C.M. and Klotz, R. 1985, Chem. Phys., 95, 213.

Mathis, J.S., Mezger, P.G. and Panagia, N. 1983, Astr. Ap., 128, 212.

Morris, M. and Jura, M. 1983, Ap. J., 264, 546.

Nee, J.B. and Lee, L.C. 1985, Ap. J., 291, 202.

Nuth, J.A. and Glicker, S. 1982, J. Quant. Spectrosc. Rad. Transf., 28, 223.

Okabe, H. 1978, Photochemistry of small molecules (Wiley, New York).

Oppenheimer, M. and Downey, C.J. 1980, Ap. J. (Letters), 241, L123.

Pineau des Forêts, G., Flower, D.R., Hartquist, T.W. and Dalgarno, A. 1986, M. N. R. A. S., submitted.

Potter, A.E. and Del Ducca, B. 1964, Icarus, 3, 103.

Pouilly, B., Robbe, J.M., Schamps, J. and Roueff, E. 1983, J. Phys. B, 16, 437.

Prasad, S.S. and Tarafdar, S.P. 1983, Ap. J., 267, 603.

Roberge, W.G., Dalgarno, A. and Flannery, B.P. 1981, Ap. J., 243, 817.

Rodgers, C.P. and Williams, A.P. 1974, J. Quant. Spectrosc. Rad. Transf., 14, 319.

Römelt, J., Peyerimhoff, S.D. and Buenker, R.J. 1978, Chem. Phys. Lett., 58, 1.

Römelt, J., Peyerimhoff, S.D. and Buenker, R.J. 1981, Chem. Phys., 54, 147.

Rostas, F. 1985, priv. comm. and in preparation.

Sandell, G. and Mattila, K. 1975, Astr. Ap., 42, 357.

Saxon, R.P. and Liu, B. 1985, ICPEAC XIV Abstracts of contributed papers, eds. M.J. Coggiola et al. (Palo Alto), p. 60.

Saxon, R.P., Lengsfield, B.H. and Liu, B. 1982, J. Chem. Phys., 78, 312.

Shih, S.-K., Peyerimhoff, S.D. and Buenker, R.J. 1977, J. Mol. Spectrosc., 64, 167; 1979, ibid. 74, 124.

Smith, P.L., Yoshino, K., Black, J.H. and Parkinson, W.H. 1980, Ap. J., 238, 874.

Solomon, P.M. 1965, as cited by Field, G.B., Somerville, W.B. and Dressler, K. 1966, Ann. Rev. Astr. Astrophys., 4, 207.

Solomon, P.M. and Klemperer, W. 1972, Ap. J., 178, 389.

Stecher, T.P. and Williams, D.A. 1967, Ap. J. (Letters), 149, L29.

Stephens, T.L. and Dalgarno, A. 1972, J. Quant. Spectrosc. Rad. Transf., 12, 569.

Suto, M. and Lee, L.C. 1985, J. Geophys. Res., 90, 13037.

van Dishoeck, E.F. 1984, Ph. D. Thesis, University of Leiden.

van Dishoeck, E.F. 1986, in preparation.

van Dishoeck, E.F. and Black, J.H. 1986, Ap. J. Suppl., in press.

van Dishoeck, E.F. and Dalgarno, A. 1983, J. Chem. Phys., 79, 873.

van Dishoeck, E.F. and Dalgarno, A. 1984a, Ap. J., 277, 576.

van Dishoeck, E.F. and Dalgarno, A. 1984b, Icarus, 59, 305.

van Dishoeck, E.F., van Hemert, M.C. and Dalgarno, A. 1982, J. Chem. Phys., 77, 3693.

Witt, A.N. and Johnson, M.W. 1973, Ap. J., 181, 363.

Wootten, A., Lichten, S.M., Sahai, R. and Wannier, P.G. 1982, Ap. J., 257, 151.

Wurm, K. 1934, Zs. für Astrophys., 8, 281; 1935, ibid. 9, 62. 


\section{DISCUSSION}

FEDERMAN: The unattenuated dissociation rate for $\mathrm{CO}$ of $7 \times 10^{-11} \mathrm{~s}^{-1}$ seems large. Is this determined under the assumption of significant self-shielding?

VAN DISHOECK: The rate assumes no self-shielding. Note that the rate listed in the table is a factor three lower than some experimental estimates.

P.A. FELDMAN: Could you comment on the ability of the Douglas mechanism, involving internal conversion to infrared photons, to effectively lengthen photodissociation lifetimes of complex molecules, particularly carbon chains?

VAN DISHOECK: The photodissociation of the long carbon chain molecules that have been observed in interstellar clouds is expected to be still very rapid. The rates for $\mathrm{HCN}$ and $\mathrm{HC}_{3} \mathrm{~N}$ are fairly well known, and the rate for $\mathrm{HC}_{3} \mathrm{~N}$ is even larger than that for $\mathrm{HCN}$, as Table 1 shows. For substantially larger molecules, internal conversion may indeed lengthen the photodissociation lifetimes, although I doubt that even the rates of complex molecules will be much smaller than about $10^{-12} \mathrm{~s}^{-1}$.

LEGER: For middle size molecules $(N \leqq 20)$, internal conversion in the molecule (i.e., electronic excitation transferring its energy to vibrations) would not protect the molecule from dissociation. The molecule becomes vibrationally very hot ( $\mathrm{T}_{\text {eff }}>3000 \mathrm{~K}$ ) and loses one atom, just as a solid loses atoms during sublimation, with a very high probability before it cools by IR emission ( $1 \mathrm{~s})$. This should prevent middle size molecules from surviving in the diffuse interstellar medium.

WALMSLEY: You mentioned briefly the question of the photodissociation rates of the isotopic species of $\mathrm{CO}$. It is of particular interest for radio astronomers to know whether there are differences between the rates of ${ }^{13} \mathrm{CO}$ and $\mathrm{C}^{18} \mathrm{O}$. Could you expand on this?

VAN DISHOECK: The calculation of the rates of isotopic species of $C O$ as function of depth in interstellar clouds is complicated by the fact that lines in the $(0,0)$ bands of isotopic species are shielded by ${ }^{12} \mathrm{CO}$, but not the lines $\left(v^{\prime}, 0\right)$ bands with $v^{\prime} \geqq 1$ due to larger isotopic shifts when a non-zero quantum number is involved. The problem is that presently it is not known exactly through which bands the photodissociation proceeds. If the photodissociation occurs mainly through $(0,0)$ band absorptions, as may well be the case, the depth dependence of the ${ }^{13} \mathrm{CO}$ and $\mathrm{C}^{18} \mathrm{O}$ rates will not be much different from that of ${ }^{12} \mathrm{CO}$. If a large fraction of the photodissociation proceeds through $v^{\prime} \neq 0,0$ bands, the depth dependence is largely determined by the self-shielding of ${ }^{13} \mathrm{CO}$ and $\mathrm{C}^{18} \mathrm{O}$, which may be different. Note however that some of the lines of these two species are very close even in $v^{\prime} \neq 0,0$ bands, so that ${ }^{13} \mathrm{Co}$ may still shield $\mathrm{c}^{18} \mathrm{O}$ in this case. 
HARTQUIST: If CO photodissociation is dominated by transitions to high Rydberg states, at what column density does self-shielding become important?

VAN DISHOECK: This depends on the oscillator strengths for the individual transitions into the higher-lying states, which are not yet well known, and on the population distribution of $\mathrm{CO}$ over the lower state rotational levels. For a typical band oscillator strength $f=10^{-2}$ and a $\mathrm{CO}$ excitation temperature of $5 \mathrm{~K}$, self-shielding becomes important at $\mathrm{CO}$ column densities of $10^{14} \mathrm{~cm}^{-2}$.

SAHAI: By looking at the sizes of ${ }^{12} \mathrm{CO}$ and ${ }^{13} \mathrm{CO}$ emission from circumstellar envelopes, one should be able to decide the relative importance of $(0,0)$ and $\left(v^{\prime}, 0\right)$ absorptions for photodissociation of $c 0$. In fact, for IRC +10216 the observed size of the ${ }^{13} \mathrm{CO}$ shell is a few times smaller than that of ${ }^{12} \mathrm{CO}$, indicating that ${ }^{13} \mathrm{CO}$ is not shielded by ${ }^{12} \mathrm{CO}$, but is self-shielded. This indicates that absorptions in the $v^{\prime} \neq 0,0$ bands are important for shielding of $\mathrm{CO}$ and its isotopes.

P.K. GHOSH: Among the molecules and molecular ions whose cross sections would be of particular importance (as pointed out by the speaker), $\mathrm{H}_{2} \mathrm{O}^{+}$and $\mathrm{HCl}^{+}$photodissociation studies should be relatively simple to study in ion traps, as these species could be generated by single step electron impact ionization.

GLASSGOLD: Do you have any estimates for the molecular ions important in interstellar clouds, such as $\mathrm{HCO}^{+}, \mathrm{HN}_{2}^{+}$, etc.?

VAN DISHOECK: To my knowledge, no experimental or theoretical information is yet available on the photodissociation of the $\mathrm{HCO}^{+}$and $\mathrm{HN}_{2}^{+}$ions. 